\title{
Companion of $\eta$ Pegasi.
}

In 1780 Sir William Herschel entered $\eta$ Pegasi as No. $2 \mathrm{I}$ of his Class VI of wide double stars, but without measures. Subsequently, in 1824 , it was observed by South, and given as No. 816 of his Catalogue, with the following mean of two measures:

$$
1824.85 \quad 33^{\circ} .9 \quad 89.82 \quad 2 \mathrm{nS} \text {. }
$$

The companion is called sextremely and sexcessively difficult $\%$, and rated from the twelfth to fifteenth magnitude, with the remark that the small star scarcely bears the slightest illumination . In looking at this star a few nights since with the 36 inch refractor, I saw that the Herschel companion was a close double, and have since made the following measures :

\begin{tabular}{|c|c|c|c|c|c|c|c|c|}
\hline & $B$ anc & C. & & \multicolumn{5}{|c|}{$A$ and $B C$. } \\
\hline 1889.526 & $83: 2$ & 0.33 & Io, Io & 1889.526 & $339^{\circ} \circ$ & $90 . " 27$ & 3 , & - \\
\hline 534 & $85 \cdot 5$ & 0.26 & Io, IO & 534 & 339.2 & 90.43 & - , & - \\
\hline 537 & 82.9 & 0.27 & IO, IO & 537 & 339.0 & 90.39 & - & -- \\
\hline-540 & 81.7 & $-0 . \underline{3}^{\mathrm{r}}$ & $10.5,10.5$ & 540 & 338.9 & 90.44 & $\overline{-}$ & $\cdots$ \\
\hline I 880.53 & $83 \cdot 3$ & 0.29 & $10.1,10.1$ & 1889.53 & 339.0 & 90.38 & & \\
\hline
\end{tabular}

The change from proper motion in the interval of 64.7 years would increase the distance of the companion from the large star a little more than 2", so that if the first measures are substantially correct, it would seem that the two stars have the same movement in space. This is hardly likely to be the case, and it is more probable that South's distance is too large.

The close pair is difficult, and can hardly fail to be a physical system. While it is not a test for the large telescope, it will not be seen with any small instrument.
M: Hamilton i 889 July 29.
S. W. Burnham.

\section{Elemente und Ephemeride für die Wiederkehr des Brorsen'schen Cometen. Von Dr. E. Lamp.}

Die nachstehenden Elemente sind aus den Beob. ' achtungen der Jahre 1873 und 1879 nach neuer und strenger Berechnung der schon fruher von Herrn Professor R. Schulze nahe richtig gefundenen Störungen durch Jupiter, Saturn, Venus, Erde und Mars abgeleitet worden.

$$
\begin{aligned}
& \text { I } 879 \text { März } 30.0 \text { M. Z. Jerlin. } \\
& M=359^{\circ} 53^{\prime} 47^{\prime \prime} 3^{8} \\
& \omega)=14 \quad 55 \quad 33.23 \\
& \delta ?=101 \mathrm{1} 847.61 \\
& i=29237.01 \\
& \varphi=54 \quad 4 \quad 49.24 \\
& \mu=649.49321
\end{aligned}
$$

Für die beiden folgenden Umläufe dẻs Cometen wurden nach einer, dem von Oppolzer für den Winnecke' und damit die nachstehende Ephemeride, welche bei einer schen Cometen angewendeten Verfahren ähnlichen Methode । Verschiebung des Periheldurchgangs urn \pm I Tag um die dic nachstehenden provisorischen, für die betr. Perihelien geltenden, Werthe der Jupiterstörungen gefunden.

$\begin{array}{lll} & 1879-1884 & 1884-1890 \\ \Delta M & +1479.71 & +98.45 \\ \Delta \omega & -\quad 4.11 & +5.94 \\ \Delta S 3 & +\quad 33.46 & -13.03 \\ \Delta i & +190.80 & -142.64 \\ \Delta \varphi & +295.73 & -118.96 \\ \Delta u & +1.5701 & -0.6940\end{array}$

I)ie neuen Elemente lauten demnach:

$$
\begin{aligned}
& T=1890 \text { Febr. } 24.135^{8} \text { M. Z. Berlin } \\
& (1)=14^{\circ} 55^{\prime} 35.89 \\
& \delta=1012733.74 \\
& i=292348.25 \\
& \varphi=54.446 .19 \\
& \mu=650.3693
\end{aligned}
$$

'Sie ergeben die heliocentrischen Aequatoreal-Coordinaten:

$$
\begin{aligned}
& x=[9.94282] r \cdot \sin (v+208.026) \\
& y=[9.98512] r \cdot \sin (v+126.426) \\
& z=[9.73684] r \cdot \sin (v+60.570)
\end{aligned}
$$

folgeuden Beträge $z u$ variiren ist:

$\begin{array}{rll}1889 & \Delta \alpha & \Delta \delta \\ \text { Nov.12 } & \mp 31^{\text {s }} & \pm 6 \\ \text { Dec. } 6 & \mp 21 & \pm 5 \\ 30 & \mp 18 & \pm 0\end{array}$

Nähere Ausführungen über meine Arbeit werden später veröffentlicht werden. 
Ephemeride für $12^{\text {h }}$ M. Z. Berlin.

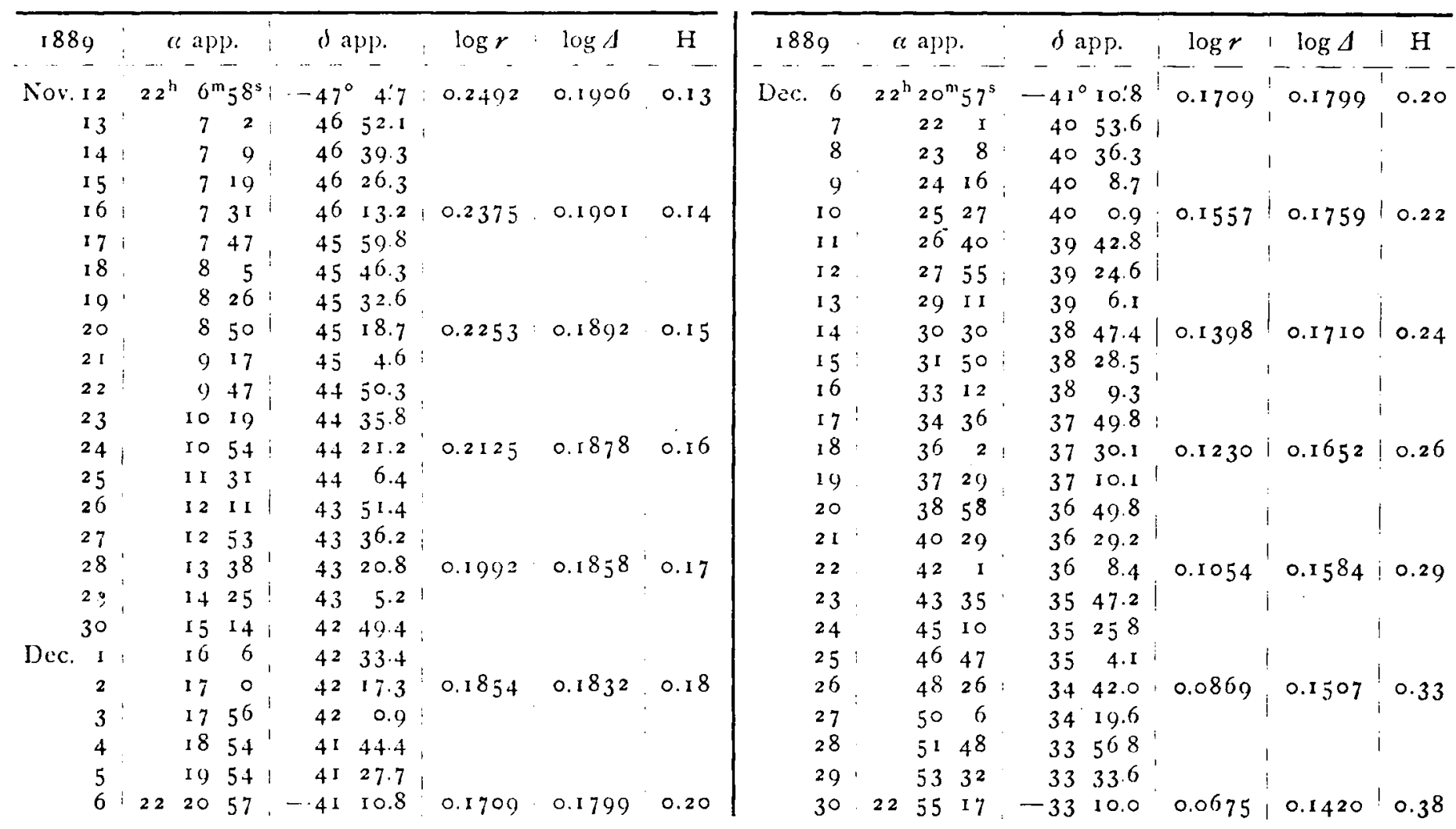

Für die Beurtheilung der Helligkeit habe ich unter $\mathrm{H}$ die Werthe von $\mathbf{1}: \boldsymbol{r}^{2} \boldsymbol{d}^{2}$ angesetzt. Dieselben betrugen 0.12 zur Zcit der Auffindung des Cometen durch Tempel in Arcetri am 14.Jan. I879 und o.62 zur Zeit der ersten Beobachtung in Windsor N. S. Wales 1879 Febr. 26.

Der Anfang der obigen Ephemeride wurde den Sternwarten der südlichen Halbkugel durch Telegramn der Centralstelle mitgetheilt.

Kiel 1889 Oct. 30 .

E. T.amp.

\section{Elliptische Elemente des Cometen 1889 III (Barnard Juni 23).}

Folgende Mittheilung über den Versuch einer Bahn- die Iängenfehler Null, so blieben in den mittleren Breiten bestimmung des Cometen 1889 III (Barnard Juni 23) ist noch Fehler von einer Minute übrig. Die Bahn scheint vielleicht von einigem Interesse.

Aus 5 Oertern 1889 Juni 25,30 , Juli 5, ro und Aug. I burg und München), Juli ro (Lick Obs.) und Aug. I (Wien, wollte ich nämlich eine Parabel rechnen; es gelang aber; bestätigt durch Juli 3 I Lick Obs.) folgende Elemente ernicht, Längen und Breiten gleichzeitig darzustellen. IVaren halten:

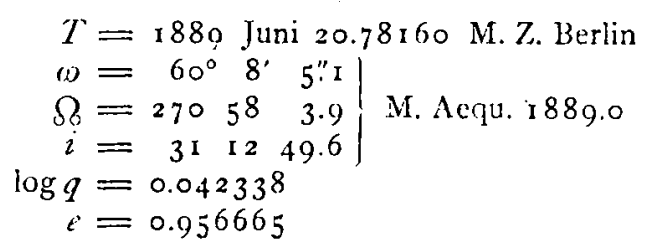

Die Umlaufszeit, die aber noch einer beträchtlichen

Frühere Erscheinungen werden sich wohl kaum nachUnsicherheit unterliegt, würde hiernach i28.3 Jahre sein. i weisen lassen wegen der Lichtschwäche des Cometen. Bei der mässigen Neigung und der Iage des absteigenden Knotens kann die Cometenbahn starke Störungen durch Jupiter erleiden; eben dieser, Umstand machte auch von vornherein die Ellipticität wahrscheinlich.

Ich gedenke den Cometen definitiv zu berechnen und bitte etwa noch unpublicirte Beobachtungen baldigst in den Astr. Nachr. mittheilen zu wollen. 